\title{
Design and Stability Analysis of a Robust Impedance Control System for a Robot Manipulator
}

\author{
Mohammad Reza Soltanpour ${ }^{1}$, Seyed Ehsan Shafiei ${ }^{2}$ \\ ${ }^{1}$ Shahid Sattari Air University, \\ Shamshiri Street, Tehran, 1599964511, Iran, \\ e-mail: m_r_soltanpour@yahoo.com \\ ${ }^{2}$ Shahrood University of Technology, \\ Daneshgah Street, Shahrood, 3619995161, Iran, \\ e-mail: sehshf@yahoo.com
}

\begin{abstract}
This paper presents a novel impedance control approach for a robot manipulator and analyzes its stability. In order to achieve desired impedance for the robot manipulator subject to applied force on the end-effector, a hybrid position/force control in the task space is developed. For this purpose, the both cases of known and unknown bounds of uncertainties are considered to design the nonlinear robust controller. It is proven that the closed loop control system shows global exponential stability under known bounds of uncertainties. In the second case, an adaptive controller is used to estimate the bounds of uncertainties. It is then proven that the closed loop system has a global asymptotically stability. The case study is a two-link elbow manipulator which is simulated. The simulation results confirm good performances of proposed control approaches.
\end{abstract}

Keywords: Robot Manipulator, Uncertainties, Robust Impedance Control, Adaptive Control.

\section{Introduction}

In addition of moving through free space, industrial robots involve their environments while operating special tasks such as assembling, polishing, deburring, pushing and power-assisting [1-5]. So, the force and position control must put on simultaneously. In spite of that, in the most researches in position control of robots it is supposed that the robot does not involve to operation environment and significantly, small position error, fast response and practical implementation are concerned in the controller design. In such control systems, a simple contact with working surface may lead notable problems; because the system dynamics change and in consequence, the closed loop system stability is not guaranteed anymore.

Hybrid position/force control and impedance control are the most noticeable methods that used to control of robots which involve their environments [6-7]. In the hybrid control, the task space is partitioned into two distinct position and force subspaces by selection matrix $S$, such that the position control is accomplished in the position space, and force control is accomplished in the force space. Despite of considering distinguish between position and force control in the hybrid method, the desired impedance of robot manipulator and dynamical behavior of environmental reaction force are not taken into account.
In the impedance control one can control the environment reaction through the endeffector path as well as position control at the same time and further, there is no need to selection matrix $S$. The main drawback of this method is that it employs identical design parameters for both position and force control. As a result, the controller performance in dealing with dynamical behavior of environment reaction force and end-effector position is the same [7].

After presenting these approaches, researchers focused their studies on concurrent position/force control. Hybrid impedance control was proposed based on the concepts of the internal and external control loops where the position and force control achieved simultaneously by using of the exact model of system dynamics [8]. Although, the access of exact dynamical model assumption is not fulfilled in the presence of structured and unstructured uncertainties as load variation, friction, disturbance and un-modeled dynamics and on the other hand, these uncertainties produce restrictions in measurement techniques. The uncertainties affect on controller performances and closed loop system stability and, in much cases cause the closed loop system becomes unstable.

Adaptive control has been used to overcome parametric uncertainties in the dynamical model of robot manipulator and to achieve 
desired impedance [9-10]. By noting to the result of these studies it can be seen that adaptive control has effective performance against parametric uncertainties but these are only parts of all uncertainties in a real system. Another method which is proposed to deal with structured and unstructured uncertainties in impedance control of robot manipulator is sliding mode control [11]. The control law is designed such that the position is controlled when the robot is in free space and the force is controlled when the robot involves. This control law is very simple and in order to avoid discontinuity of control input, the system could have only uniformly bounded stability. In [12] the desired impedance attained based on relation between position tracking error and force tracking error by employing sliding mode control. Stability analysis shows that the closed loop system has uniformly bounded stability. Additionally, there are other effective works in the field of robust impedance control [1316]. But these controllers are designed based on the dynamical model of robot manipulator in task space and therefore the computation magnitudes are high and they need fast processors in implementation phase. It is worth mentioning that the fuzzy method is also used to model-free impedance control of robot for quick tasks [17].

\section{Robot Dynamics in Joint Space}

Dynamical equation of an n-link robot manipulator is as follows [18]:

$$
\begin{aligned}
& M(q) \ddot{q}+V_{m}(q, \dot{q}) \dot{q}+G(q)+F_{d} \dot{q}+F_{s}(\dot{q}) \\
& +T_{d}+\tau_{e}=\tau(t)
\end{aligned}
$$

Where $q(t), \dot{q}(t)$ and $\ddot{q}(t)$ are $n \times 1$ vector of position, velocity and angular acceleration of robot joints, respectively. $M(q)$ is $n \times n$ inertia matrix, $V_{m}(q, \dot{q}) \dot{q}$ is $n \times 1$ vector including Coriolis and centrifugal forces and $G(q)$ is $n \times 1$ gravity vector. Also, $F_{d}$ denotes $n \times n$ diagonal matrix of dynamic friction and $F_{S}$ denotes $n \times 1$ vector of static friction. Finally, $T_{d}, \tau_{e}$ and $\tau(t)$ are $n \times 1$ vector of disturbance and un-modeled dynamics, environment torque and joint input torque, respectively. The relation (1) has following properties [19].

\section{Property 1.}

The inertia matrix of $M(q)$ is symmetric positive definite and for any $q \in R^{n}$ is uniformly bounded as

$$
\mu_{1} I \leq M(q) \leq \mu_{2} I \quad \text { or } \quad \mu_{1} \leq\|M(q)\| \leq \mu_{2}
$$

Where $\|\bullet\|$ denotes the two norm and $\mu_{1}$ and $\mu_{2}$ are positive constants.

\section{Property 2.}

The matrix of $\dot{M}(q)-2 V_{m}(q, \dot{q})$ is skewsymmetry.

$$
y^{T} \dot{M}(q) y=2 y^{T} V_{m}(q, \dot{q}) y \quad, \quad \forall y, q, \dot{q} \in R^{n}
$$

\section{Property 3}

In equation (1) the matrices $M(q)$ and $V_{m}(q, \dot{q})$, and the gravity vector $G(q)$ are linear in the parameters of $P=\left[\begin{array}{llll}P_{1} & P_{2} & \ldots & P_{m}\end{array}\right]^{T}$. So, it concludes:

$$
M(q) \ddot{q}+V_{m}(q, \dot{q}) \dot{q}+G(q)=W(q, \dot{q}, \ddot{q}) p
$$

Where $p$ is $m \times 1$ vector of robot manipulator parameters and $W(q, \dot{q}, \ddot{q})$ is $n \times m$ matrix including known functions of the position, velocity and acceleration of joints which is called regression matrix. In most applications, the end-effector path is determined in task space and following relation is used to map it into joint space [20-22].

$$
X=h(q)
$$

In which, $X$ is $n \times 1$ position vector of endeffector and $h(q)$ presents nonlinear transmission function from task space to joint space. The end-effector velocity in task space is expressed by

$$
\dot{X}=J(q) \dot{q}
$$

Where $J(q)$ is $n \times n$ Jacobian matrix and $\dot{X}$ is $n \times 1$ vector of end-effector velocity in task space. thus,

$\dot{q}=J(q)^{-1} \dot{X}$

Where $J(q)^{-1}$ indicates the inverse of Jacobian matrix. For validation of equation (7), the desired trajectory in task space must design in such a way that it be smooth and does not pass from the singular points of 
robot manipulator. At this condition, the inverse of Jacobian matrix is derivative and has full rank throughout the trajectory. derivating of (6) respect to time, gives:

$$
\ddot{X}=J(q) \ddot{q}+\dot{J}(q) \dot{q}
$$

Then, the joint acceleration is obtained as:

$$
\ddot{q}=J(q)^{-1} \ddot{X}-J(q)^{-1} \dot{J}(q) \dot{q}
$$

For transferring control vector from task space into joint space, one can use[22]:

$$
\tau(t)=J^{T}(q) f(t)
$$

Where $f(t)$ is $n \times 1$ force vector in task space.

\subsection{Robot dynamics in task space}

In most investigations, the task space model is employed to impedance control plan [1113] which is achieved from (1), (7), (9) and (10) as:

$$
\left\{\begin{aligned}
M_{x}(q) \ddot{X}+V_{m_{x}}(q, \dot{q}) \dot{X}+N_{x}(q, \dot{q})+F_{e}=F \\
M_{x}(q)=J(q)^{-T} M(q) J(q)^{-1} \\
V_{m_{x}}(q, \dot{q})=J(q)^{-T}\left(V_{m}-M(q) J(q)^{-1} \dot{J}(q)\right. \\
\left.\quad+F_{d}\right) J(q)^{-1} \\
N_{x}(q, \dot{q})=J(q)^{-T} G(q)+J(q)^{-T} F_{s}(\dot{q}) \\
+J(q)^{-T} T_{d}
\end{aligned}\right.
$$

\subsection{Desired impedance of robot manipulator}

Since, the chief goal of impedance control method is the dynamical behavior control of robot manipulator one should define a desired impedance for robot manipulator as control object and then find a relation between acceleration in task space, desired impedance and environment reaction to aim it [7-8]. So

$$
\begin{aligned}
& \ddot{X}=\frac{d}{d t}\left(\dot{X}_{d}-\Gamma^{-1}\left\{Z_{m}^{-1}(S)\right\} * F_{e}\right) \\
& Z_{m}(S)=M S+B+\frac{K}{S}
\end{aligned}
$$

in which, $\dot{X}_{d}$ is $n \times 1$ vector of desired velocity in task space, $Z_{m}(s)$ shows the Laplace transform of desired impedance, the sign of $*$ denotes convolution operator, $S$ is Laplace transform variable, $\Gamma^{-1}$ indicates inverse Laplace transform and finally, $M, B$ and $K$ ar e $n \times n$ diagonal constant matrix. As stated by conventional method in robust impedance control, a controller is designed such that the relation (12) remains valid [78]. Eliminating known dynamics is one of the preferred approaches which utilized for designing controllers. But according to equation (11), it can be found that using task space model leads to tremendous computation amount. So, implementing these kinds of controllers needs high speed processors. On the other hand, these controllers accompany computational errors as a result of computing position, velocity and acceleration from equations (5), (6) and (8). Succeeding section presents a strategy to overcome these difficulties.

\section{Transforming Impedance Control into Position/Force Control}

Taking Laplace transform from (12) yields:

$$
S Z_{m}(S)=\frac{F_{e}(S)}{X_{d}(S)-X(S)}
$$

Thus the relation between position and force in the task space is obtained as:

$$
X_{d}-X=\Gamma^{-1}\left\{\frac{1}{S} Z_{m}^{-1}(S)\right\} * F_{e}
$$

Now, if one designs the position tracking system such that $X$ could track $\tilde{X}_{d}$ with following equation,

$$
\tilde{X}_{d}=X_{d}-\Gamma^{-1}\left\{\frac{1}{S} Z_{m}^{-1}(S)\right\} * F_{e}
$$

Then in exact tracking, it concludes $\tilde{X}_{d}=X$ and consequently (14) would be valid and desired impedance of robot manipulator would be achieved. Therefore, the necessary condition that realizes this goal is

$\left\|\widetilde{X}_{d}-X\right\|=0$

In the presence of uncertainties, satisfying (16) contribute to success provided that the tracking need to be fast or in the other words the robust position/force tracking controller out to be designed in a way that it guarantees the global asymptotic stability of closed loop system in a short period of time.

$$
\lim _{t \rightarrow \infty}\left\|\widetilde{X}_{d}-X\right\|=0
$$


An assumption should be considered in robust controller design is that the new trajectory need to be bounded. According (15) and the facts that the desired trajectory in task space $\left(X_{d}\right)$ is bounded and also, from passivity of workspace design and boundedness of $F_{e}$, it is concluded that this assumption is always valid. Thus, designing robust impedance controller is converted to designing robust position/force tracking controller in task space.

\section{Robust Position/Force Tracking in Task Space}

In order to design robust controller consider following assumptions

$$
\begin{aligned}
& \left\|F_{d} y+F_{S}(y)\right\|=\xi_{f_{0}}+\xi_{f_{1}}\|y\|, \forall y \in R^{n} \\
& \left\|T_{d}\right\| \leq \xi_{t} \\
& \|\widetilde{p}\|=\|p-\hat{p}\| \leq \rho
\end{aligned}
$$

Where $\xi_{f_{0}}, \xi_{f_{1}}, \xi_{t}$ and $\rho$ are positive constant and $\hat{P}$ is $m \times 1$ vector of the estimation of robot manipulator parameters. The sliding surface is defined in task space as follows:

$$
\begin{aligned}
& \dot{X}_{p}=\alpha\left(\widetilde{X}_{d}-X\right)+\dot{\widetilde{X}}_{d} \\
& S_{X}=\dot{X}_{p}-\dot{X}=\alpha\left(\tilde{X}_{d}-X\right)+\left(\dot{\tilde{X}}_{d}-\dot{X}\right)
\end{aligned}
$$

Where $\alpha$ is a positive constant and $S_{X}$ denotes $n \times 1$ vector of sliding surface in task space. Establishing position error as $\widetilde{X}_{d}-X=e$ and velocity error as $\dot{\widetilde{X}}_{d}-\dot{X}=\dot{e}$, yields

$$
S_{X}=\alpha e+\dot{e}
$$

Equation (7) is used to transfer $\dot{X}_{p}$ into joint space as:

$\dot{q}_{p}=J^{-1}(q) \dot{X}_{p}$

Differentiating (21) respect to time gives:

$\ddot{q}_{p}=J^{-1}(q) \ddot{X}_{p}+\dot{J}^{-1}(q) \dot{X}_{p}$

Sliding surface is defined in joint space as:

$$
S_{q}=\dot{q}_{p}-\dot{q}
$$

By multiplying Jacobian matrix in both sides of above relation and using (6) and (19) yields

$$
J(q) S_{q}=J(q) \dot{q}_{p}-J(q) \dot{q}=\dot{X}_{p}-\dot{X}=S_{X}
$$

Sliding surface in task space is associated to one in joint space by equation below.

$S_{q}=J^{-1}(q) S_{X}$

For transferring control vector from task space into joint space one may act as follows [23].

$$
\tau(t)=J^{-1}(q) f(t)
$$

According to (17), the robust control vector is defined as:

$$
\begin{aligned}
\tau(t)= & \hat{M}(q) \ddot{q}_{p}+\hat{V}_{m}(q, \dot{q}) \dot{q}_{p}+\hat{G}(q) \\
& +J^{-1}(q) \hat{F}_{e}+\gamma \hat{M}(q) J^{-1}(q) S_{X} \\
& +u+u_{r}
\end{aligned}
$$

Where $\hat{M}(q), \quad \hat{V}_{m}(q, \dot{q}) \quad$ and $\quad \hat{G}(q) \quad$ are estimations of $M(q), V_{m}(q, \dot{q})$ and $G(q)$ respectively. $\hat{F}_{e}$ denotes estimation of environment reaction force, $\gamma$ is positive constant and $u$ and $u_{r}$ are new control vectors. It should be noted that $\hat{F}_{e}$ is added to robust control vector by a force sensor. Since the sliding surface $S_{q}$ is associated with the sliding surface $S_{X}$ by (25), then (27) could be expressed as:

$$
\begin{aligned}
\tau(t)= & \hat{M}(q) \ddot{q}_{p}+\hat{V}_{m}(q, \dot{q}) \dot{q}_{p}+\hat{G}(q) \\
& +J^{-1}(q) \hat{F}_{e}+\gamma \hat{M}(q) S_{q}+u+u_{r}
\end{aligned}
$$

Substituting above equation into (1) and noting that $\Delta A=F_{d} \dot{q}+F_{S}(q)+T_{d}$, give

$$
\begin{aligned}
& M(q) \ddot{q}+V_{m}(q, \dot{q}) \dot{q}+G(q)+\tau_{e}+\Delta A= \\
& \hat{M}(q) \ddot{q}_{p}+\hat{V}_{m}(q, \dot{q}) \dot{q}_{p}+\hat{G}(q)+J^{-1}(q) \hat{F}_{e} \\
& +\gamma \hat{M}(q) S_{q}+u+u_{r}
\end{aligned}
$$

From (23), (26) and (29) concludes

$$
\begin{aligned}
& M(q) \ddot{q}+V_{m}(q, \dot{q}) \dot{q}+G(q)+\Delta A= \\
& \hat{M}(q)\left(\dot{S}_{q}+\ddot{q}\right)+\hat{V}_{m}(q, \dot{q})\left(S_{q}+\dot{q}\right) \\
& +\hat{G}(q)+\gamma \hat{M}(q) S_{q}+u+u_{r}
\end{aligned}
$$

Simplifying (30) yields 


$$
\begin{aligned}
& \hat{M}(q) \dot{S}_{q}+\hat{V}_{m}(q, \dot{q}) S_{q}=(M(q)-\hat{M}(q)) \ddot{q} \\
& +\left(V_{m}(q, \dot{q})-\hat{V}_{m}(q, \dot{q})\right) \dot{q}+(G(q)-\hat{G}(q)) \\
& -\gamma \hat{M}(q) S_{q}+\left(\Delta A-u_{r}\right)-u
\end{aligned}
$$

Using (4), equation (31) can be shown by

$$
\begin{gathered}
\hat{M}(q) \dot{S}_{q}+\hat{V}_{m}(q, \dot{q}) S_{q}=W(q, \dot{q}, \ddot{q})(P-\hat{P}) \\
-\gamma \hat{M}(q) S_{q}+\left(\Delta A-u_{r}\right)-u
\end{gathered}
$$

Above relation is simplified by defining parameter error $P-\hat{P}=\widetilde{P}$ as follows:

$$
\begin{aligned}
& \hat{M}(q) \dot{S}_{q}+\hat{V}_{m}(q, \dot{q}) S_{q}=W(q, \dot{q}, \ddot{q}) \widetilde{P} \\
&-\gamma \hat{M}(q) S_{q}+\left(\Delta A-u_{r}\right)-u
\end{aligned}
$$

\subsection{Stability proof}

Following Lyapunov function candidate is introduced for providing the stability proof of the closed loop system.

$$
V(t)=\frac{1}{2} S_{q}^{T} \hat{M}(q) S_{q}
$$

Time derivative of (34) is

$$
\dot{V}(t)=S_{q}^{T} \hat{M}(q) \dot{S}_{q}+\frac{1}{2} S_{q}^{T} \dot{\hat{M}}(q) S_{q}
$$

Substituting (33) into (35) gives

$$
\begin{aligned}
\dot{V}(t) & =S_{q}^{T}\left(\begin{array}{l}
-\hat{V}_{m}(q, \dot{q}) S_{q}+W(q, \dot{q}, \ddot{q}) \tilde{P} \\
-\gamma \hat{M}(q) S_{q}+\left(\Delta A-u_{r}\right)-u
\end{array}\right) \\
& +\frac{1}{2} S_{q}^{T} \dot{\hat{M}}(q) S_{q}
\end{aligned}
$$

From property 2 , one can have

$$
\begin{aligned}
\dot{V}(t)= & -2 \gamma V+S_{q}^{T} W(q, \dot{q}, \ddot{q}) \widetilde{P}+S_{q}^{T}\left(\Delta A-u_{r}\right) \\
& -S_{q}^{T} u
\end{aligned}
$$

Since $\quad \Delta A=F_{d} \dot{q}+F_{s}(q)+T_{d}$, by using assumptions (1) and (2) from section 4,one may have:

$$
\begin{aligned}
& \|\Delta A\| \leq \xi_{f_{0}}+\xi_{f_{1}}\|\dot{q}\|+\xi_{t} \\
& \eta=\xi_{f_{0}}+\xi_{f_{1}}\|\dot{q}\|+\xi_{t}
\end{aligned}
$$

Now, $u$ and $u_{r}$ are defined as:

$$
u=\left\{\begin{array}{cc}
\frac{W W^{T} S_{q} \rho^{2}}{\left\|S_{q}^{T} W\right\| \rho+\varepsilon\left\|S_{q}\right\| e^{-\beta\left(t-t_{0}\right)}} & \left\|S_{q}\right\| \neq 0 \\
0 & \left\|S_{q}\right\|=0
\end{array}\right.
$$

Where $\varepsilon, \lambda$ and $\beta$ are positive constant. Putting (39) into (37) and simplification yields

$$
\begin{aligned}
\dot{V}(t) & \leq-2 \gamma V+\frac{\|Z\| \lambda e^{-\beta\left(t-t_{0}\right)}}{\|Z\|+\lambda e^{-\beta\left(t-t_{0}\right)}} \\
& +\frac{\varepsilon\left\|S_{q}\right\| S_{q}^{T} W \| \rho e^{-\beta\left(t-t_{0}\right)}}{\left\|S_{q}^{T} W\right\| \rho+\varepsilon\left\|S_{q}\right\| e^{-\beta\left(t-t_{0}\right)}}
\end{aligned}
$$

Whereas

$0 \leq \frac{X Y}{X+Y} \leq Y \quad \forall X, Y$

One concludes from (40) and (41) that

$\dot{V}(t) \leq-2 \gamma V+\left(\lambda+\varepsilon\left\|S_{q}\right\|\right) e^{-\beta\left(t-t_{0}\right)}$

In the above equation the exponential term converges to zero when time goes to infinity and so it is resulted that $\dot{V} \leq 0$. So, the closed loop system with the proposed controllers has global asymptotic stability.

\subsection{Global exponential stability proof}

The scalar function $\varphi(t)$ is introduced as:

$\varphi(t)=\dot{V}(t)+2 \gamma V(t)-\left(\lambda+\varepsilon\left\|S_{q}\right\|\right) e^{-\beta\left(t-t_{0}\right)}$

Now, the Lyapunov function is obtained by coming equation.

$$
\begin{aligned}
& V(t)=V\left(t_{0}\right) e^{-2 \gamma\left(t-t_{0}\right)}+ \\
& \int_{t_{0}}^{t} e^{-2 \gamma(t-Q)}\left[\left(\lambda+\varepsilon\left\|S_{q}\right\|\right) e^{-\beta\left(Q-t_{0}\right)}+\varphi(Q)\right] d Q
\end{aligned}
$$

From (42) and (43) it deduces that $\varphi(t) \leq 0$. So,

$$
\begin{aligned}
& V(t) \leq V\left(t_{0}\right) e^{-2 \gamma\left(t-t_{0}\right)} \\
& +\left(\lambda+\varepsilon\left\|S_{q}\right\|\right) \int_{t_{0}}^{t} e^{-2 \gamma(t-Q)} e^{-\beta\left(Q-t_{0}\right)} d Q
\end{aligned}
$$

and after simplification,

$$
\begin{aligned}
& V(t) \leq V\left(t_{0}\right) e^{-2 \gamma\left(t-t_{0}\right)} \\
& +\frac{\left(\lambda+\varepsilon\left\|S_{q}\right\|\right)}{2 \gamma-\beta}\left(e^{-\beta\left(t-t_{0}\right)}-e^{-2 \gamma\left(t-t_{0}\right)}\right)
\end{aligned}
$$

According to relations (2) and (46) and continuity of $\dot{V}(t)$ and it's convergence to zero one can use Barbalat's Lemma [24] to show that the closed loop system with the proposed controllers is globally exponentially stable for any initial conditions and any initial time. The overall robust controller is provided in below. 


$$
\begin{aligned}
& \tau(t)=\hat{M}(q) \ddot{q}_{p}+\hat{V}_{m}(q, \dot{q}) \dot{q}_{p}+\hat{G}(q) \\
& +J^{-1}(q) \hat{F}_{e}+\gamma \hat{M}(q) J^{-1}(q) S_{X}+u+u_{r} \\
& u=\left\{\begin{array}{cc}
\frac{W W^{T} S_{q} \rho^{2}}{\left\|S_{q}^{T} W\right\| \rho+\varepsilon\left\|S_{q}\right\| e^{-\beta\left(t-t_{0}\right)}} & \left\|S_{q}\right\| \neq 0 \\
0 & \left\|S_{q}\right\|=0
\end{array}\right. \\
& u_{r}=\left\{\begin{array}{c}
\frac{Z \eta}{\left(\|Z\|+\lambda e^{-\beta\left(t-t_{0}\right)}\right)} \\
Z=\eta S_{q}
\end{array}\right.
\end{aligned}
$$

\section{Adaptive Robust Controller}

As mentioned in the previous section, the structure of robust control is depended on determining of bounded functions. The bound of uncertainties must be therefore known for designing this kind of controllers. In the most robotic applications, bounds of parametric uncertainties (e.g. load variations) are known; but if the certain application of a robot is not determined then recognizing bounds of the unstructured uncertainties is very difficult and making a mistake in evaluating them and choosing them large unnecessarily leads to design a high gain robust controller and it in itself may cause saturating of actuators. Whereas, the dynamics of the unstructured uncertainties is approximately specified, according to assumptions in section 4 , and only their parameters are unknown, this problem could be solved by incorporating an adaptive controller into the robust control part. Briefly speaking, the adaptive controller estimates these parameter bounds, and the input gain of this adaptive robust controller is therefore specified by these estimated parameter bounds. So, according to assumptions of section 4, it results from equation (38) that

$\eta=W_{1}^{T}(\dot{q}) \phi$

Where $W_{1}^{T}(\dot{q})$ is $1 \times l$ vector of known functions and $\phi$ is $l \times 1$ vector of unknown parameters. Consequently, these parameters can be estimated by incorporating adaptive control into robust control of section 4. For this purpose, equation (47) is changed to

$$
\begin{aligned}
& \tau(t)=\hat{M}(q) \ddot{q}_{p}+\hat{V}_{m}(q, \dot{q}) \dot{q}_{p}+\hat{G}(q) \\
& +J^{-1}(q) \hat{F}_{e}+\gamma \hat{M}(q) J^{-1}(q) S_{X}+u+u_{r} \\
& u=\left\{\begin{array}{cc}
\frac{W W^{T} S_{q} \rho^{2}}{\left\|S_{q}^{T} W\right\| \rho+\varepsilon\left\|S_{q}\right\| e^{-\beta\left(t-t_{0}\right)}} & \left\|S_{q}\right\| \neq 0 \\
0 & \left\|S_{q}\right\|=0
\end{array}\right. \\
& u_{r}=\left\{\begin{array}{c}
\hat{Z} \hat{\eta} \\
\left.\|\hat{Z}\|+\lambda e^{-\beta\left(t-t_{0}\right)}\right) \\
\hat{Z}=\hat{\eta} S_{q}
\end{array}\right.
\end{aligned}
$$

Where $\hat{Z}$ and $\hat{\eta}$ are estimations of $Z$ and $\eta$, respectively.

\subsection{Stability analysis}

The estimation error of the unstructured uncertainty parameters is defined as $\phi-\hat{\phi}=\widetilde{\phi}$. By employing the relation (50) as a Lyapunov function candidate, one may obtain:

$$
\begin{aligned}
& V(t)=\frac{1}{2} S_{q}^{T} \hat{M}(q) S_{q}+\frac{1}{2} \widetilde{\phi}^{T} \tilde{\phi} \\
& \dot{V}(t)=S_{q}^{T} \hat{M}(q) \dot{S}_{q}+\frac{1}{2} S_{q}^{T} \dot{M}(q) S_{q}-\widetilde{\phi}^{T} \dot{\hat{\phi}}
\end{aligned}
$$

From (31), yields:

$$
\begin{aligned}
\dot{V}(t) & =-\gamma S_{q}^{T} \hat{M}(q) S_{q}+S_{q}^{T} W(q, \dot{q}, \ddot{q}) \widetilde{P} \\
& +S_{q}^{T}\left(\Delta A-u_{r}\right)-S_{q}^{T} u-\widetilde{\phi}^{T} \dot{\hat{\phi}}
\end{aligned}
$$

Using assumptions of the section 4 and relations (38), (49) and (52) yields

$$
\begin{aligned}
& \dot{V}(t) \leq-\gamma S_{q}^{T} \hat{M}(q) S_{q}+\left\|S_{q}^{T} W(q, \dot{q}, \ddot{q})\right\| \rho \\
& +(\|Z\|-\|\hat{Z}\|)+\|\hat{Z}\|-S_{q}^{T} u_{r}-S_{q}^{T} u-\widetilde{\phi}^{T} \dot{\hat{\phi}}
\end{aligned}
$$

Substituting $u$ and $u_{r}$ from (49) into (53) gives

$$
\begin{aligned}
& \dot{V}(t) \leq-\gamma S_{q}^{T} \hat{M}(q) S_{q}+\frac{\|Z\| \lambda e^{-\beta\left(t-t_{0}\right)}}{\|Z\|+\lambda e^{-\beta\left(t-t_{0}\right)}}+ \\
& \frac{\varepsilon\left\|S_{q}\right\| S_{q}^{T} W \| \rho e^{-\beta\left(t-t_{0}\right)}}{\left\|S_{q}^{T} W\right\| \rho+\varepsilon\left\|S_{q}\right\| e^{-\beta\left(t-t_{0}\right)}}+\left\|S_{q}\right\| W_{1}^{T} \widetilde{\phi}-\widetilde{\phi}^{T} \dot{\hat{\phi}}
\end{aligned}
$$

From (41) and (54) concludes

$$
\begin{aligned}
\dot{V}(t) & \leq-\gamma S_{q}^{T} \hat{M}(q) S_{q}+\left(\lambda+\varepsilon\left\|S_{q}\right\|\right) e^{-\beta\left(t-t_{0}\right)} \\
& +\left\|S_{q}\right\| W_{1}^{T} \widetilde{\phi}-\widetilde{\phi}^{T} \dot{\hat{\phi}}
\end{aligned}
$$

The exponential term of the above equation converges to zero. If one choose 


$$
\dot{\hat{\phi}}=W_{1}\left\|S_{q}\right\|
$$

Then $\dot{V}(t) \leq 0$. So $S_{q}$ and in consequence from (24), $S_{X}$ converges to zero. Thus the closed loop system with the proposed controllers has global asymptotic stability. The comprehensive adaptive robust controller is provided in below.

$$
\begin{aligned}
& \tau(t)=\hat{M}(q) \ddot{q}_{p}+\hat{V}_{m}(q, \dot{q}) \dot{q}_{p}+\hat{G}(q) \\
& +J^{-1}(q) \hat{F}_{e}+\gamma \hat{M}(q) J^{-1}(q) S_{X}+u+u_{r} \\
& u=\left\{\begin{array}{cc}
\frac{W W^{T} S_{q} \rho^{2}}{\left\|S_{q}^{T} W\right\| \rho+\varepsilon\left\|S_{q}\right\| e^{-\beta\left(t-t_{0}\right)}} & \left\|S_{q}\right\| \neq 0 \\
0 & \left\|S_{q}\right\|=0
\end{array}\right. \\
& u_{r}=\left\{\begin{array}{c}
\hat{Z} \hat{\eta} \\
\left.\|\hat{Z}\|+\lambda e^{-\beta\left(t-t_{0}\right)}\right) \\
\hat{Z}=\hat{\eta} S_{q}
\end{array}\right. \\
& \hat{\eta}=W_{1}^{T} \hat{\phi} \quad, \dot{\hat{\phi}}=W_{1}\left\|S_{q}\right\|
\end{aligned}
$$

\section{Discussion}

In the most of robot applications a trajectory is considered in task space for tracking of end-effector position. On the other hand, the controllers are designed in joint space; for this reason there is required to task space trajectory converted to joint space one via inverse kinematics. However, this conversion goes with errors in the presence of uncertainties and because all feedbacks are in joint space, these errors are not observable and so can't be modified [19-23]. Accordingly, in this paper the proposed controllers have been designed in task space and also in view of the fact that the joint space model is employed for designing these controllers instead of the task space model. From (11), one can result that the control signal computations are reduced significantly. Note that according to (14), the environment reaction force will be zero when the manipulator is not involved the surface and the position/force control is hence reduced to just position control. Therefore, versus other position/force controllers this control strategy does not need to change for achieving position control exclusively.

\section{Simulation Results}

A two-link elbow robot depicted in Fig. 1 is considered as a case study here. Dynamical equation of this robot is given by [25]:

$$
\begin{gathered}
{\left[\begin{array}{ll}
M_{11} & M_{12} \\
M_{21} & M_{22}
\end{array}\right]\left[\begin{array}{l}
\ddot{q}_{1} \\
\ddot{q}_{2}
\end{array}\right]+\left[\begin{array}{l}
h_{1}(q, \dot{q}) \\
h_{2}(q, \dot{q})
\end{array}\right]=\left[\begin{array}{l}
u_{1}(t) \\
u_{2}(t)
\end{array}\right]} \\
M_{11}=\left(l_{2}{ }^{2} m_{2}+2 l_{1} l_{2} \cos \left(q_{2}\right)+l_{1}{ }^{2}\left(m_{1}+m_{2}\right)\right) \\
M_{12}=M_{21}=\left(l_{2}{ }^{2} m_{2}+l_{1} l_{2} m_{2} \cos \left(q_{1}\right)\right) \\
M_{22}=l_{2}{ }^{2} m_{2} \\
h_{1}(q, \dot{q})=-m_{2} l_{1} l_{2} \sin \left(q_{2}\right) \dot{q}_{2}{ }^{2} \\
\quad-2 m_{2} l_{1} l_{2} \sin \left(q_{2}\right) \dot{q}_{1} \dot{q}_{2} \\
+m_{2} l_{2} g \cos \left(q_{1}+q_{2}\right) \\
+\left(m_{1}+m_{2}\right) l_{1} g \cos \left(q_{1}\right) \\
+F_{d_{1}} \dot{q}_{1}+F_{s_{1}}\left(\dot{q}_{1}\right)+T_{d_{1}} \\
h_{2}(q, \dot{q})=\left(m_{2} l_{2} g \cos \left(q_{1}+q_{2}\right)\right) \\
+\left(m_{2} l_{1} l_{2} \sin \left(q_{2}\right) \dot{q}_{1}^{2}\right) \\
+F_{d_{2}} \dot{q}_{2}+F_{s_{2}}\left(\dot{q}_{2}\right)+T_{d_{2}}
\end{gathered}
$$

Where $l_{i}$ and $m_{i}$ are the length and the mass of the $i$-th joint, respectively. $g$ denotes gravity acceleration, $F_{S}$ is static friction and $T_{d}$ indicates disturbance and un-modeled dynamics. Also $u_{i}$ is the input torques of the $i$-th joint. Robot parameters are provided in table 1 and controller parameters and regression matrix elements are given in tables 2 and 3 , respectively.

For adaptive controller design one may choose the physical parameters as follows:

$$
\begin{aligned}
\hat{P}= & {\left[\begin{array}{lll}
l_{u_{2}}^{2} m_{u_{2}} & l_{u_{1}} l_{u_{2}} m_{u_{2}} l_{u_{1}}^{2}\left(m_{u_{1}}+m_{u_{2}}\right) \\
& m_{u_{2}} l_{u_{2}} g_{u} & \left(m_{u_{1}}+m_{u_{2}}\right) l_{u_{1}} g_{u}
\end{array}\right]^{T} }
\end{aligned}
$$

$l_{u}, m_{u}$ and $g_{u}$ are unknown part of $l, m$ and $g$, respectively. The performance of the position controller and position/force controller are evaluated separately. In inspecting of position control it is supposed that the robot moves in free space. Desired trajectory and initial conditions for this free movement are given in table 4 . 


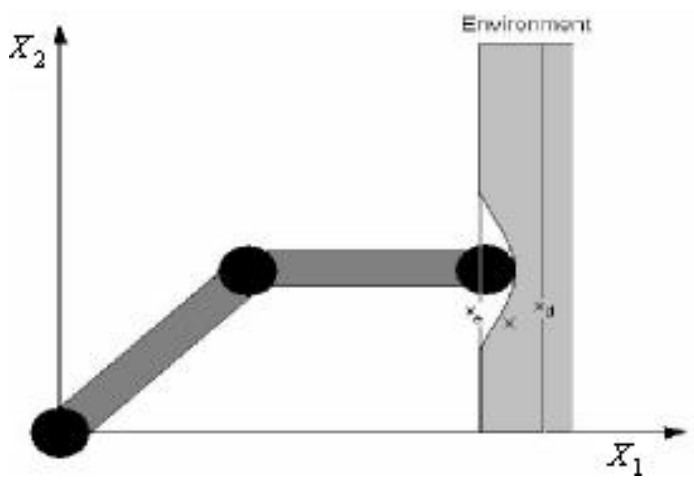

Figure 1. Tow-link Elbow Robot Manipulator

Table1. Robot Manipulator Parameters

\begin{tabular}{|c|c|}
\hline$l_{1}=0.38 \mathrm{~m}$ & $l_{2}=0.29 \mathrm{~m}$ \\
\hline$m_{1}=1.4 \mathrm{~kg}$ & $m_{2}=0.8 \mathrm{~kg}$ \\
\hline$g=9.8$ & $T_{d_{1}}=T_{d_{2}}=0.5$ \\
\hline$F_{d_{1}}=F_{d_{2}}=0.5$ & $F_{s_{1}}\left(\dot{q}_{1}\right)=F_{s_{2}}\left(\dot{q}_{2}\right)=0.5$ \\
\hline
\end{tabular}

Table 2. Controller Parameters

\begin{tabular}{|l|l|l|}
\hline$\hat{l}_{1}=0.48 \quad \mathrm{~m}$ & $\hat{l}_{2}=0.4 \quad \mathrm{~m}$ & $M=I$ \\
\hline$\hat{m}_{1}=1.5 \mathrm{~kg}$ & $\hat{m}_{2}=0.9 \mathrm{~kg}$ & $K=100 I$ \\
\hline$\beta=0.01$ & $\gamma=100$ & $B=60 I$ \\
\hline$\varepsilon=0.5$ & $\alpha=250$ & $\lambda=15$ \\
\hline$\hat{g}=9$ & $\rho=0.1$ & $\eta=2$ \\
\hline
\end{tabular}

Table 3. Regression Matrix

\begin{tabular}{|c|c|}
\hline \multicolumn{2}{|c|}{$W_{11}=\ddot{q}_{r_{1}}+\ddot{q}_{r_{2}}$} \\
$W_{12}=2 \cos \left(q_{2}\right) \ddot{q}_{r_{1}}+\cos \left(q_{2}\right) \ddot{q}_{r_{2}}$ \\
$-\sin \left(q_{2}\right) \dot{q}_{2} \dot{q}_{r_{2}}-2 \sin \left(q_{2}\right) \dot{q}_{1} \dot{q}_{r_{2}}$ \\
\hline$W_{13}=\ddot{q}_{r_{1}}$ & $W_{14}=\cos \left(q_{1}+q_{2}\right)$ \\
\hline$W_{15}=\cos \left(q_{1}\right)$ & $W_{21}=\ddot{q}_{r_{1}}+\ddot{q}_{r_{2}}$ \\
\hline $\begin{array}{l}W_{22}=\cos \left(q_{2}\right) \ddot{q}_{r_{1}} \\
+\sin \left(q_{2}\right) \dot{q}_{1} \dot{q}_{r_{1}}\end{array}$ & $W_{23}=0$ \\
\hline$W_{24}=\cos \left(q_{1}+q_{2}\right)$ & $W_{25}=0$ \\
\hline
\end{tabular}

Table 4. Desired Trajectory and Initial Conditions in Free Space

\begin{tabular}{|c|c|}
\hline$X_{d_{1}}=0.0284$ & $X_{d_{2}}=0.0403$ \\
$+0.005 \sin (3 t)$ & $+0.005 \cos (3 t)$ \\
\hline$X_{1}(0)=0.0255$ & $X_{2}(0)=0.048$ \\
\hline
\end{tabular}

In order to evaluate the position/force control, the environment, a vertical wall, is modelled as a pure stiffness with no friction in Figure
1. Desired trajectory and initial conditions are provided in table 5 for this case.

Table 5. Desired Trajectory and Initial Conditions in Position/ Force Control

\begin{tabular}{|c|c|}
\hline$X_{d_{1}}=0.26$ & $X_{d_{2}}=0.403+0.05 \cos (3 t)$ \\
\hline$X_{1}(0)=0.2$ & $X_{2}(0)=0.48$ \\
\hline
\end{tabular}

In this case, relation between position $X_{1}$ and the environment reaction in direction of $X_{1}$ is given by

$$
X_{d_{1}}(S)-X_{1}(S)=\frac{F_{e_{1}}(S)}{S^{2}+60 S+100}
$$

Also one will have $F_{e_{2}}=0$ since the working environment is modeled as mentioned before. In the simulation it is assumed $X_{e_{1}}=0.25$

Centimetre and the wall is modeled as a spring with following equation [24].

$$
F_{e_{1}}=1000\left(X_{1}-0.25\right)
$$

According to desired trajectory given in table 5 and relation (15) the beneath trajectory should be tracked.

$$
\tilde{X}_{d}=\left[X_{d_{1}}-\Gamma^{-1}\left(\frac{1}{S^{2}+60 S+100}\right) * F_{e_{1}} \quad X_{d_{2}}\right]^{T}
$$

Desired force $F_{e d_{1}}$ in direction of $X_{1}$ is equal to 5 Newtons.

Simulation 1 - In this case, the robust controller (47) is simulated for position control in free space. As shown in figures 2 and 3 , despite of presence of structured and unstructured uncertainties, this controller operates well and tracking errors converge zero after around 0.5 seconds. According to Figures 2 and 3, maximum tracking errors are 28 and $30 \mathrm{~mm}$, respectively.

Simulation 2 - In this case, the adaptive robust controller (57) is simulated for position control in free space. This controller operates well too and can drive tracking errors to converge zero as depicted in figures 4 and 5. Maximum tracking error is $25 \mathrm{~mm}$ and estimated unstructure uncertainty is 2.5 as shown in Figure 6.

Simulation 3 - In this case, the robust position/force control (47) is simulated for achieving desired impedance. As can be found from Figures 7 and 8, tracking of $X_{d_{1}}$ 
and $X_{d_{2}}$ is obtained in a short duration of time. Figure 9 shows the acceptable tracking of the desired force $F_{e d_{1}}$.

Simulation 4 - In this case, the adaptive robust position/force control (57) is simulated for achieving desired impedance. Figures 10 and 11 verify the satisfactory tracking of $X_{d_{1}}$ and $X_{d_{2}}$ in a small time. The environment reaction force, the wall here, is depicted in Figure 12. By noting the reasonable tracking of force and position it can be concluded that the desired impedance of robot manipulator is realized.

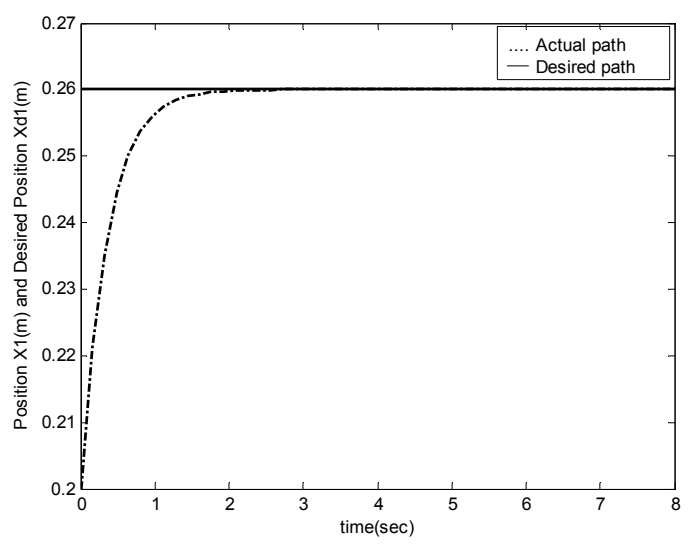

Figure 2. Tracking Error $X_{d_{1}}$

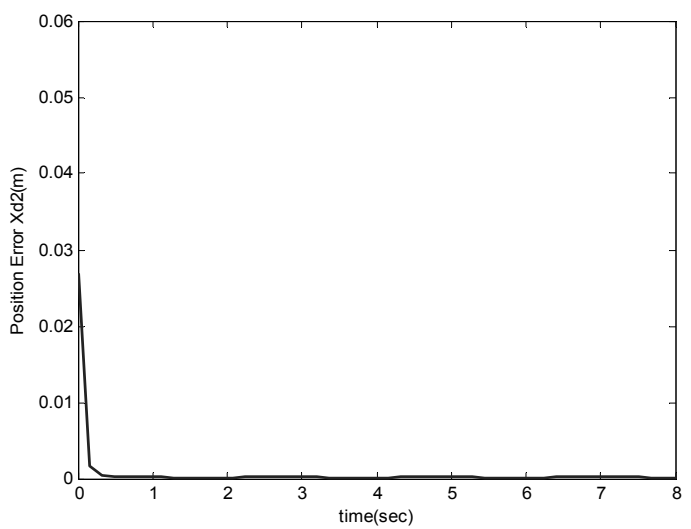

Figure 3. Tracking Error $X_{d_{2}}$

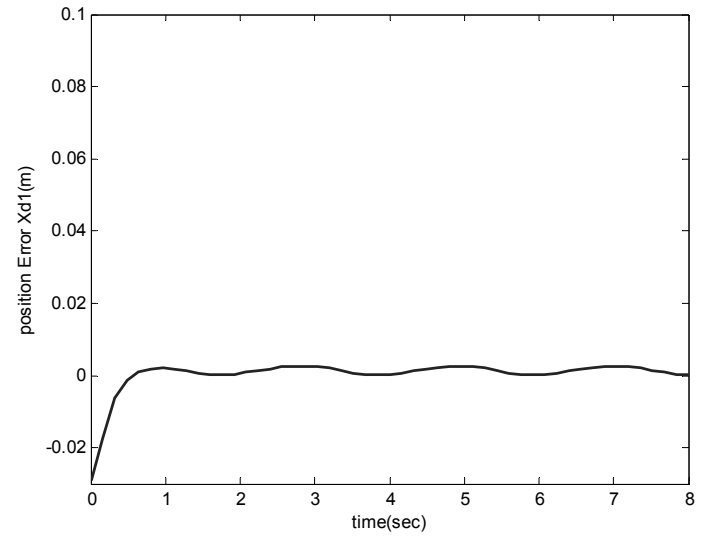

Figure 4. Tracking Error $X_{d_{1}}$

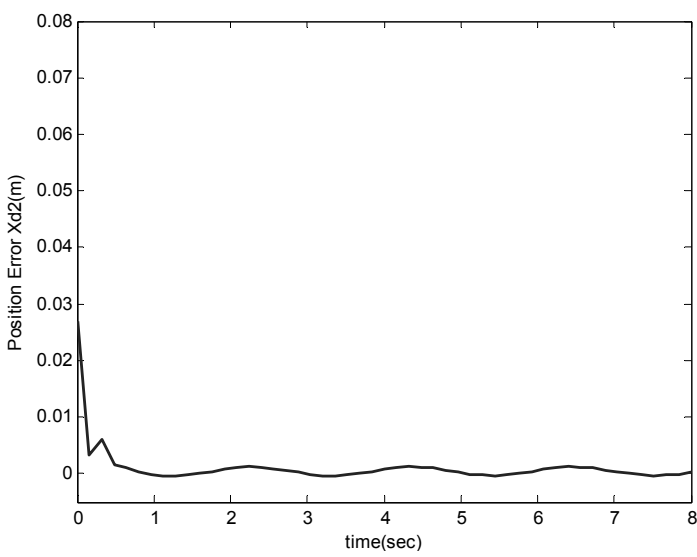

Figure 5. Tracking Error $X_{d_{2}}$

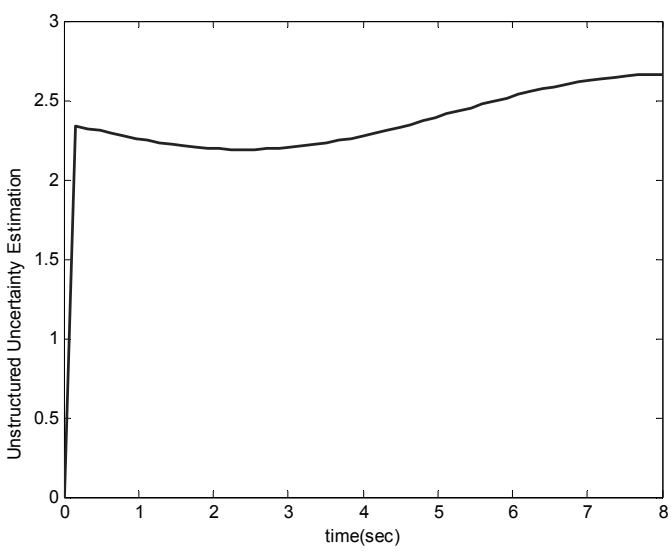

Figure 6. Estimated Unstructure Uncertainty 


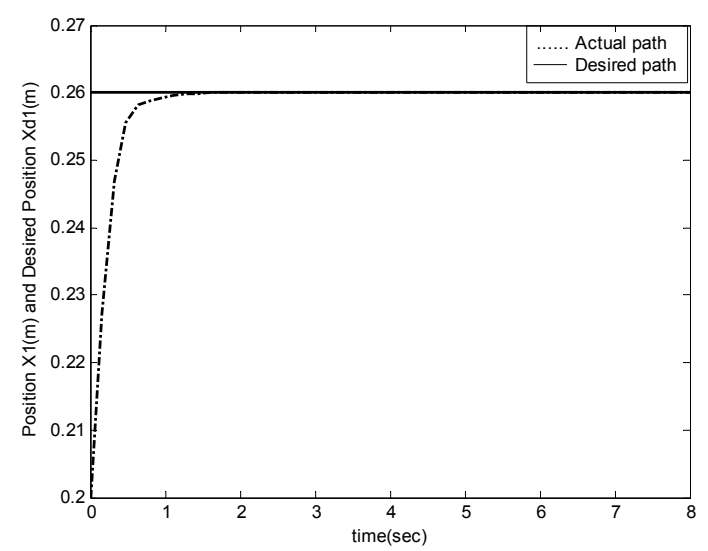

Figure 7. Tracking $X_{d_{1}}$

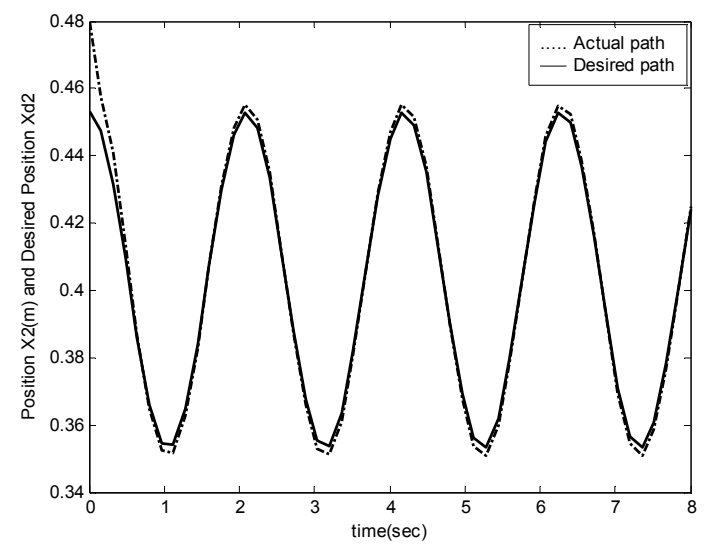

Figure 8. Tracking $X_{d_{2}}$

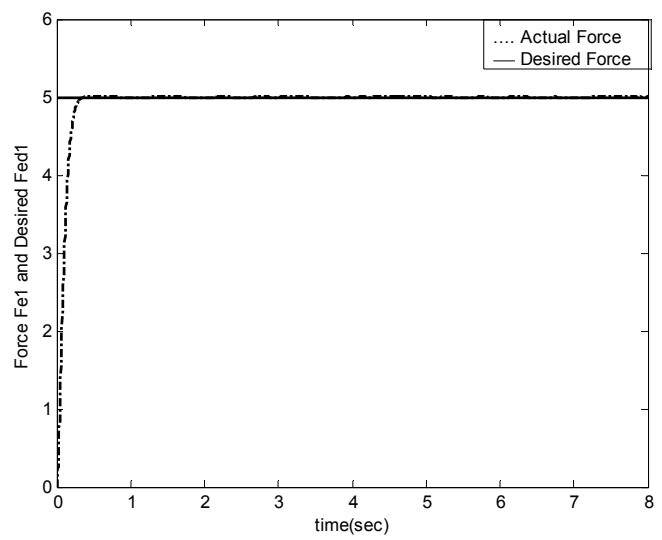

Figure 9. Tracking of Environment Reaction Force $F_{e d_{1}}$

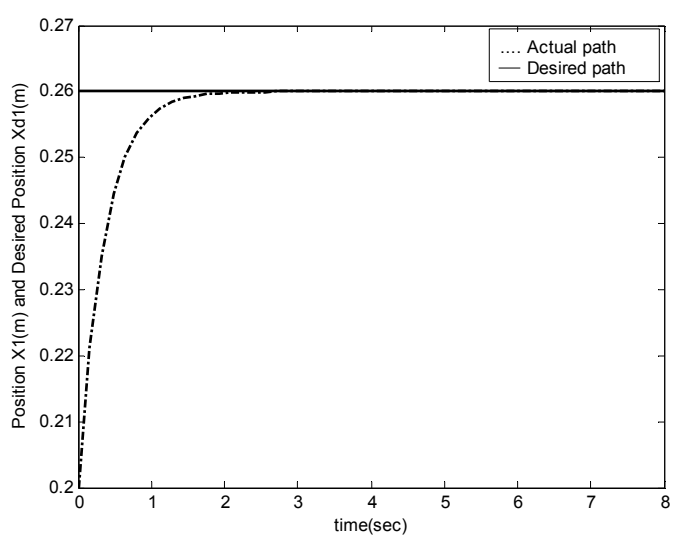

Figure 10. Tracking $X_{d_{1}}$

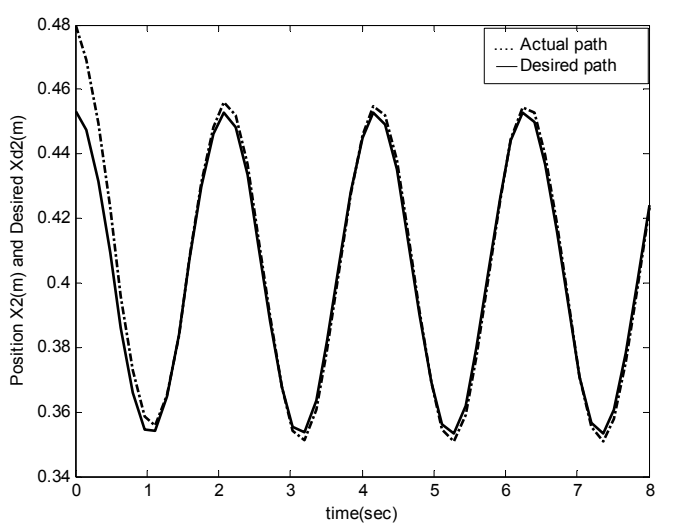

Figure 11. Tracking $X_{d_{2}}$

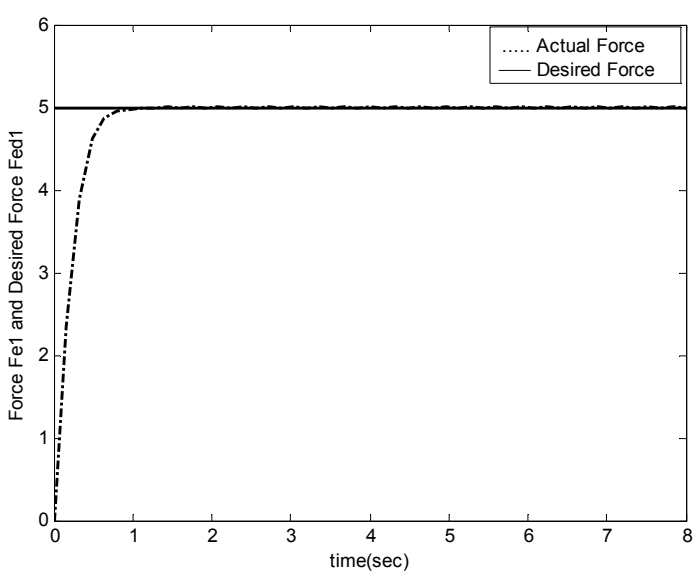

Figure 12. Tracking of Environment Reaction Force $F_{e d_{1}}$ 


\section{Conclusion}

In this paper, in order to design robust impedance control, a new trajectory was defined for tracking control of robot in task space based on dynamical behavior of environment reaction force and desired impedance of robot manipulator and also the necessary condition for converting robust impedance control into robust position/force tracking control was developed. Subsequently, a robust nonlinear tracking controller was proposed by considering the bounds of structured and unstructured uncertainties that can satisfy this condition. It is proved that the closed loop system with the proposed controller has global exponential stability. Since the bounds of structured uncertainties as load variations are known but the parameter bounds of unstructured uncertainties remain unknown in the most robot applications, an adaptive controller was incorporated into robust controller for estimating those bounds real time. It is also proved that the closed loop system with adaptive robust controller has global asymptotic stability. The trajectory tracking and the desired impedance of robot manipulator and consequently the satisfactory dynamical behavior of environment reaction were achieved. A prominent advantage of the proposed controllers is that in situation which robot operates in free space and doesn't involve the surface, the position tracking is obtained and there is not required to change control system. In the other words, the proposed controllers have the ability of position/force control and position control simultaneously. Mathematical analysis and simulation results justified the efficient performance of the controllers.

\section{REFERENCES}

1. CHAN, S. P., H. C. LIAW, Generalized Impedance Control of Robot for Assembly Tasks Requiring Compliant Manipulation, IEEE Transactions Industrial Electronics, Vol. 43, No. 4, 1996, pp. 453-461.

2. BASANEZ, L., J. ROSELl, Robotic Polishing Systems, IEEE Transactions on Robotics and Automation, Vol. 12, No. 3, 2005, pp. 35-43.
3. KAZEROONI, H., Automated Robotic Deburring Using Impedance Control, IEEE Control Systems, Vol. 8, No. 1, 1988, pp. 21-25.

4. KATSURA, S., J. SUZUKI, K. OHNISHI, Pushing Operation by Flexible Manipulator Taking Environmental Information into Account, IEEE Transactions Industrial Electronics, Vol. 53, No. 5, 2006, pp. 1688-1697.

5. HARA, S., A Smooth Switching from Power-assist Control to Automatic Transfer Control and Its Application to a Transfer Machine, IEEE Transactions Industrial Electronics, Vol. 54, No. 1, 2007, pp. 638-650.

6. RAIBERT, M. H., J. J. CRAIG, Hybrid Position/Force Control of Manipulators, ASME Journal of Dynamic System, Measurement and Control, Vol. 102, 1981, pp. 126-133.

7. HOGAN, N. H., Impedance Control: An Approach to Manipulation: Part ITheory, Part II - Implementation, Part III - Application, ASME Journal of Dynamic System, Measurement and Control, Vol. 107, 1985, pp. 1-24.

8. ANDERSON, R., M. W. SPONG, Hybrid Impedance Control of Robotic Manipulators, IEEE Journal on robotic and Automation, Vol. 4, No. 5, 1988, pp. 1073-1080.

9. KELLY, R., R. CARELLI, M. AMESTEGUI, R. ORTEGA, Adaptive Impedance Control of Robot Manipulators, International Journal on robotic and Automation, Vol. 4, No. 3, 1989, pp. 134-141.

10. JUNG, S., T. C. HSIA, Stability and Convergence Analysis of Robust Adaptive Force Tracking Impedance Control of Robot Manipulators, Proceedings of IEEE International Conference on Intelligent Robots and Systems, 1999, pp. 635-640.

11. HACE, A., K. JEZERNIK, S. URAN, Robust Impedance Control, Proceedings of IEEE International Conference on Control Applications, 1998, pp. 583-587. 
12. CHAN, S. P., Implementation of Generalized Impedance Control for Robot Manipulators, Proceedings of IEEE International Conference on Control Applications, 1999, pp. 418-423.

13. JUNG, S., T. C. HSIA, R. G. BONITZ, Force Tracking Impedance Control of Robot Manipulators under Unknown Environment, IEEE Transactions on Control Systems Technology, Vol. 12, No. 3, 2004, pp. 474-483.

14. WANG, H., K. H. LOW, M. Y. WANG, Combined Impedance/Direct Control of Robot Manipulators, Proceedings of IEEE/RSJ International Conference on Intelligent Robots and Systems, 2006, pp. 3605-3610.

15. LIPPIELLO, V., B. SICILIANO, L. VILLANI, A Position-Based Visual Impedance Control for Robot Manipulators, IEEE International Conference on Robotics and Automation, 2007, pp. 2068-2073.

16. LEE, E., J. PARK, K. A. LOPARO, C. B. SCHRADER, P. H. CHANG, BangBang Impact Control Using Hybrid Impedance/Time-Delay Control, IEEE / ASME Transactions on Mechatronics, Vol. 8, No. 2, 2003, pp. 272-277.

17. FATEH, M. M., Fuzzy Impedance Control to Perform Quick Robotic Tasks, Int. Cong. on Manufacturing Eng., Tehran, Iran, Dec. 12-15, 2005.

18. QU, Z., D. DAWSON, Robust Tracking Control of Robot Manipulators, 1st Edition, IEEE Press, 1996.
19. DIXON, W. E., Adaptive Regulation of Amplitude Limited Robot Manipulators with Uncertain Kinematics and Dynamics, in Proceedings of Amer. Control Conf., 2004, pp. 3844-3939.

20. CHEAH, C. C., C. LIU, J. J. E. SLOTINE, Approximate Jacobian Adaptive Control for Robot Manipulators, in Proceedings IEEE International Conference of Robotics Automation, 2004, pp. 3075-3080.

21. CHEAH, C. C., C. LIU, J. J. E. SLOTINE, Adaptive Jacobian Tracking Control of Robots Based on Visual Task-space Information, in Proceedings IEEE International Conference of Robotics Automation, 2005, pp. 3509-3514.

22. CHEAH, C. C., C. LIU, J. J. E. SLOTINE, Adaptive Jacobian Tracking Control of Robots With Uncertainties in Kinematic, Dynamic and Actuator Models, IEEE Transaction on Automatic Control, Vol. 51, No. 6, June, 2006.

23. CHEAH, C. C., H. C. LIAW, Inverse Jacobian Regulator with Gravity Compensation: Stability and Experiment, IEEE Transaction on Automatic Control, Vol. 21, No. 4, August 2005.

24. SPONG, M., M.VIDYASAGAR, Robot Dynamics and Control, 1st Edition, Wiley, 2006. 